Trauma Berufskrankh 2018 20 (Suppl 4):S244-S250 https://doi.org/10.1007/s10039-018-0366-y Online publiziert: 17. April 2018 (c) Springer Medizin Verlag GmbH, ein Teil von Springer Nature 2018 CrossMark

\section{Scholtysik' $\cdot$ M. Wich ${ }^{2,3,4}$}

${ }^{1}$ Spitzenverband der gewerblichen Berufsgenossenschaften und der Unfallversicherungsträger der öffentlichen Hand, Deutsche Gesetzliche Unfallversicherung (DGUV), Berlin, Deutschland

${ }^{2}$ Klinik für Unfallchirurgie und Orthopädie, BG Klinikum Unfallkrankenhaus Berlin gGmbH, Berlin, Deutschland

${ }^{3}$ Achenbach-Krankenhaus Königs Wusterhausen, Königs Wusterhausen, Deutschland ${ }^{4}$ Wissenschaftlicher Beirat, Fachgesellschaft für interdisziplinäre, medizinische Begutachtung (FGIMB), Hamburg, Deutschland

\title{
Minderung der Erwerbsfähigkeit (MdE) in der gesetzlichen Unfallversicherung
}

\section{Aktuelle Diskussion und Ergebnisse aus der MdE-Expertengruppe}

\section{Ausgangslage}

In der Sozialgerichtsbarkeit, den medizinischen Fachgesellschaften und auch innerhalb der gesetzlichen Unfallversicherung wurde zuletzt verstärkt diskutiert, ob die Erfahrungswerte für die Minderung der Erwerbsfähigkeit (MdE; sog. MdE-Tabellen) noch dem aktuellen wissenschaftlichen Stand entsprechen. Kritisiert werden zudem mangelnde Transparenz und fehlende Begründungen, sodass kaum nachvollziehbar sei, auf welcher Grundlage die MdE-Einschätzungen beruhen und ob die heutigen Anforderungen des allgemeinen Arbeitsmarktes und der aktuelle medizinischtechnische Entwicklungsstand (z.B. bei der Prothesenversorgung) berücksichtigt werden. Der Spitzenverband der Deutschen Gesetzlichen Unfallversicherung (DGUV) hat deshalb die Initiative für eine Revision der MdE-Erfahrungswerte ergriffen und ein ehrenamtlich tätiges Gremium multiprofessioneller Experten einberufen. Diese „MdE-Expertengruppe" hat ihre Beratungen im Sommer 2015 aufgenommen und die Arbeiten Anfang 2018 abgeschlossen. Nach systematischer Überprüfung der wichtigsten MdE-Werte bei Amputationen werden nunmehr neue MdEEckwerte vorgeschlagen.

\section{Entwicklung und Wesen der MdE}

Die MdE ist ein wesentlicher Maßstab für die Höhe der Verletztenrente nach einem Arbeits- oder Wegeunfall oder einer anerkannten Berufskrankheit. Das SGB (Sozialgesetzbuch) VII definiert diese in $\$ 56$ Abs. 2 wie folgt:

Die Minderung der Erwerbsfähigkeit richtet sich nach dem Umfang der sich aus der Beeinträchtigung des körperlichen und geistigen Leistungsvermögens ergebenden verminderten Arbeitsmöglichkeiten auf dem gesamten Gebiet des Erwerbslebens.

Für die Bemessung der MdE ist aber nicht der Gesundheitsschaden als solcher entscheidend, sondern der Funktionsverlust unter medizinischen, juristischen, sozialen und wirtschaftlichen Gesichtspunkten (BSG vom 22.06.2004 -B2 U 14/03 R-). Es gilt das Prinzip des sog. „abstrakten Schadensausgleichs“, d.h. bei gleichen Unfallfolgen ist unabhängig von deren Auswirkungen auf die konkret ausgeübte Tätigkeit die MdE gleich hoch einzuschätzen. Da die Unfallrente keinen zivilrechtlichen Schadensersatzanspruch darstellt und das Ausmaß der durch einen Unfall verursachten MdE erfahrungsgemäß nicht völlig genau feststellbar ist (vgl. BSG vom 21.03.1974 -8/2 RU
55/72-; BSG vom 20.12.2016 -B $2 \mathrm{U}$ 11/15 R-), hat das Bundessozialgericht (BSG) in zahlreichen Entscheidungen festgestellt, dass die MdE durch Schätzung festgestellt werden darf und sich nach allgemeinen Erfahrungssätzen ausrichtet (BSG vom 02.05.2001 -B2 U 24/00 R-; BSG vom 20.12.2016 -B 2 U 11/15R-). Die entsprechenden „MdE-Tabellen“ sind bisher von den UV(Unfallversicherungs)-Trägern und Gerichten in der Rechtspraxis als wirklichkeits- und maßstabsgerecht und somit auch als sozial adäquat anerkannt.

Das BSG hat zuletzt nochmals die Bedeutung dieser Tabellenwerke hervorgehoben. Wichtig sei aber, dass diese dem aktuellen medizinisch-wissenschaftlichen Erkenntnisstand folgen, d.h. einer zumindest überwiegend vertretenen Auffassung, die auf breitem Konsens und Akzeptanz beruhe. Konkret hat das BSG die Herabsetzung der MdE von bisher 60 auf $50 \%$ bei Verwendung einer mikroprozessorgesteuerten Beinprothese (CLeg) abgelehnt, da bisher wissenschaftlich (ärztlich) nicht nachgewiesen sei, dass trotz bestehender Gebrauchsvorteile daraus eine entscheidende Verbesserung der Erwerbsfähigkeit resultieren würde. Dies werde in der Literatur zwar teilweise diskutiert und sei auch grundsätzlich möglich, jedoch habe sich bisher keine 
herrschende abweichende Auffassung gebildet. Das Gericht weist ferner darauf hin, dass eigentlich der Gesetzgeber aufgerufen wäre, konkrete Regeln für den Maßstab und das Verfahren von MdETabellen nach $₫ 56$ Abs. 2 SGB VII aufzustellen, ähnlich dem Verfahren nach der Versorgungsmedizinverordnung (VersMedV). In der Rechtspraxis komme den MdE-Tabellen durchaus die normative Bedeutung von Rechtssätzen im materiellen Sinne zu, was aus rechtsstaatlicher Sicht eine formelle Normsetzung durch den Gesetzgeber erfordere. Trotz verfassungsrechtlichen Bedenken sehen Nusser und Spellbrink [1] bis zu einer etwaigen neuen gesetzlichen Regelung den Weiterbestand bzw. die Legitimation der MdE-Tabellen als rechtlich notwendige Bewertungsgrundlage, um nicht das seit Jahrzehnten funktionierende System der MdE-Bemessung erheblich zu gefährden.

Die gesetzliche Unfallversicherung nahm im Reigen der deutschen Sozialversicherungen von Anfang an eine Sonderstellung ein. Zum einen war und ist diese ausschließlich durch die Arbeitgeber finanziert, zum anderen tritt die gesetzliche Unfallversicherung mit ihren Leistungen an die Stelle von Ansprüchen des verletzten Arbeiters an den Unternehmer (Ablösung der Unternehmerhaftpflicht). Prävention, Rehabilitation und am Ende auch die Entschädigung (Unfallrente) des durch einen Arbeitsunfall entstandenen Gesundheitsschadens sind dabei untrennbar miteinander verbunden. Die Rechtsprechung verweist zudem regelmäßig auf den zentralen Leitgedanken der „sozialen Befriedung“ im Arbeitsleben, der die Aufgabe der Unfallversicherung maßgebend mitprägt. In wiederkehrenden Entscheidungen wurde bestätigt, dass die Unfallrente zwar ihre (ursprüngliche) Rolle als Lohnersatzfunktion für potenzielle Erwerbseinbußen nicht verloren habe bzw. einem Schmerzensgeldanspruch nicht gleichzustellen sei. Dennoch sei ein gesellschaftlich bedingter Funktionswandel eingetreten, bei dem die Unfallrente auch bestimmt sei, dem Ausgleich immaterieller Nachteile zu dienen, wie z. B. Verletzung der körperlichen Unversehrtheit, Integritätsverluste bei Amputationen, äußerliche Entstellungen, Mehraufwand und Schmerzen (BSG vom 03.12.2002 -B2 U12/02 R-; BGH vom 03.12.2002 -VI ZR 304/01-; BSG vom 31.03.1998 -B4RA 49/96R-). Diesen Wandel der Unfallrente beschreibt schon das Bundesverfassungsgericht in einer Grundsatzentscheidung aus dem Jahr 1972. Es rechtfertigt den Ausschluss von zivilrechtlichen Schmerzensgeldansprüchen mit der gesetzgeberischen Grundentscheidung der Haftungsersetzung und der mit diesem Haftungsausschluss der Arbeitgeber verbundenen Sicherung des sog. Betriebsfriedens in den Unternehmen (BVerfG vom 07.11.1972 -1 BvL 4/71-). Durch das Prinzip der Haftungsbefreiung bzw. Haftungsersetzung durch die UV-Träger sollen Konflikte zwischen den Arbeitsvertragsparteien möglichst reduziert und gleichzeitig der Betriebsfrieden gefördert werden (BVerfG vom 07.11.1972 -1 BvL 4/71-).

Verlässliche MdE-Werte sind auch die Basis dafür, dass die UV-Träger ihrem Auftrag bestmöglicher Rehabilitation und Eingliederung der Versicherten mit allen geeigneten Mitteln nach $₫ 1$ Ziff. 2 SGB VII gerecht werden können, ohne dass dieser Prozess durch parallele Rentenstreitigkeiten bzw. falsche Begehrensvorstellungen unnötig überlagert und belastet wird. Dass die MdE-Erfahrungswerte trotz aller Kritik diesen wichtigen Zweck in der Vergangenheit erfüllt haben, wird von keiner Seite bestritten und muss auch zukünftig gewährleistet sein. So werden in der gesetzlichen Unfallversicherung von jährlich ca. 25.000 neuen Arbeitsunfall- und BK(Berufskrankheit)-Renten lediglich ca. 2,7\% gerichtlich angefochten, wobei es nur in ca. $18 \%$ dieser Fälle zu einer (teilweisen) Korrektur des Rentenbescheides kommt, d.h. in ca. 97\% aller Fälle wird die im Verwaltungsverfahren ggf. nach Einholung eines ärztlichen Gutachtens getroffene Entscheidung der UV-Träger von den Versicherten akzeptiert [2].

\section{Kritik an der Praxis der MdE- Erfahrungswerte}

Ein Großteil der MdE-Werte (insbesondere nach Amputationen) hat sich in den letzten Jahrzehnten kaum verändert, was zu Recht die Frage nach einer Revision aufwirft. Kritisiert wird, dass die MdEWerte

- nicht ausreichend begründet sind,

- den Wandel des Erwerbslebens nicht berücksichtigen,

- den medizinischen-technischen Fortschritt nicht abbilden,

- keinen Konsens der einschlägigen Fachkreise darstellen,

- widersprüchliche und uneinheitliche Bewertungen beinhalten,

- auf keinem transparenten Verfahrensprozess beruhen.

Dies aufgreifend, wird teilweise infrage gestellt, ob und in welcher Weise die zunehmend aufwendigeren Rehabilitationsmaßnahmen sowie eine verbesserte, hochwertigere Prothesenversorgung als funktionsverbesserndes Hilfsmittel nach dem Verlust von Gliedmaßen Eingang in die MdE-Bewertung finden müssen. Das Leistungsvermögen und die Arbeitsmöglichkeiten von Menschen mit Gliedmaßenverlusten seien heute erheblich verbessert [3]. Im Zusammenspiel mit wesentlichen Veränderungen der qualitativen und quantitativen Anforderungen im Erwerbsleben (u.a. dokumentiert durch die regelmäßigen BIBB[Bundesinstitut für Berufsbildung]/ BAuA[Bundesanstalt für Arbeitsschutz und Arbeitsmedizin]-Erwerbstätigenbefragungen) ergäben sich bei entsprechender prothetischer Versorgung z.T. erhebliche Herabsetzungen der MdEWerte $[4,5]$. So begründe der Verlust eines Beines im Oberschenkel heute nur noch eine MdE von $40 \%$ (bisher $70 \%$ ), im Unterschenkel 30\% anstatt bisher $40 \%$ und der Verlust der Hand eine MdE von $45 \%$ anstatt bisher $60 \%$.

\section{Entstehung der MdE- Expertengruppe}

Der entscheidende Anstoß, die MdEWerte auf den Prüfstand zu stellen, erfolgte auf dem Deutschen Sozialgerichtstag Ende 2012 [6]. Dieser hatte der DGUV empfohlen, die Federführung für einen Konsensusprozess zu übernehmen und das schwierige Thema in einer multiprofessionellen Gruppe unabhängiger 
Experten zu diskutieren und Vorschläge einer Neubewertung zu erarbeiten. Aktualisierte MdE-Werte können in der Sozialgerichtsbarkeit nur dann Akzeptanz erfahren, wenn sie auf einem breiten Konsens der einschlägigen Fachkreise und Interessengruppen beruhen und eine Vereinheitlichung der bisherigen MdE-Tabellen erreicht wird. Auch parallele Entwicklungen im Bereich des sozialen Entschädigungsrechts (Versorgungsmedizin-Verordnung), d.h. die vom zuständigen Ärztlichen Sachverständigenbeirat Versorgungsmedizin (ÄSVB) vorgenommenen Neubewertungen zum Grad der Schädigungsfolge (GdS) und Grad der Behinderung (GdB) sollten beobachtet und mit berücksichtigt werden. Beide Rechtsmaterien weisen zwar Unterschiede auf, die MdE stellt auf die „Erwerbsfähigkeit auf dem allgemeinen Arbeitsmarkt" ab, während beim GdS/GdB die „Teilhabebeeinträchtigungen in allen Lebensbereichen (einschließlich des Arbeitsmarktes)“ zu berücksichtigen sind. Es bestehen aber auch viele Gemeinsamkeiten. So erfolge z.B. die medizinische Einschätzung der Funktionseinschränkungen unabhängig vom Bewertungsmaßstab, was in der Vergangenheit dazu geführt habe, dass in beiden Rechtsbereichen die ausgewiesenen (Erfahrungs-)Werte oftmals eng beieinander lagen.

Die DGUV hat dies aufgegriffen und ein unabhängiges, neutrales und ehrenamtlich tätiges Gremium mit unfallchirurgisch-orthopädischen, reha-, sozial- und arbeitsmedizinischen Experten einberufen, die von den jeweiligen medizinischen Fachgesellschaften und Institutionen benannt wurden, ergänzt um Experten der Unfallversicherungsträger. Wissenschaftlicher Leiter und Vorsitzender der MdE-Expertengruppe ist Herr Prof. Dr. Marcus Schiltenwolf (Leiter der Arbeitsgemeinschaft „Sozialmedizin und Begutachtungsfragen in der Deutschen Gesellschaft für Orthopädie und Unfallchirurgie“). Die Projektleitung hat Herr Dirk Scholtysik, Leiter des für die Begutachtung zuständigen Referates der DGUV.

Trauma Berufskrankh 2018 · 20 (Suppl 4):S244-S250

https://doi.org/10.1007/s10039-018-0366-y

๑ Springer Medizin Verlag GmbH, ein Teil von Springer Nature 2018

\section{Scholtysik $\cdot$ M. Wich}

\section{Minderung der Erwerbsfähigkeit (MdE) in der gesetzlichen Unfallversicherung. Aktuelle Diskussion und Ergebnisse aus der MdE-Expertengruppe}

\section{Zusammenfassung}

Die Expertengruppe unter der Schirmherrschaft der DGUV (Deutsche Gesetzliche Unfallversicherung) hat die MdE(Minderung der Erwerbsfähigkeit)-Werte nach Gliedmaßenverlusten in einem interdisziplinären methodischen Ansatz umfassend, systematisch und wissenschaftlich überprüft. Grundlage der neuen Vorschläge sind die Gesundheits- bzw. Funktionsstörungen in Anlehnung an die ICF (International Classification of Functioning, Disability and Health) und die sich daraus ergebende Einteilung in vergleichbare Funktionsstörungsklassen. Untersucht wurde, von welchen Einschränkungen am Arbeitsmarkt für Versicherte mit unfallbedingten Funktionseinschränkungen heute auszugehen ist. Dazu wurden eine rehabilitationswissenschaftliche Literaturrecherche und eine Befragung der maßgeblichen arbeitswissenschaftlichen Institute der Bundesrepublik Deutschland durchgeführt. Im Ergebnis konnten daraus jedoch keine validen Arbeitsmarktdaten zu verschlossenen Beschäftigungsmöglichkeiten generiert werden. Um ihrem Auftrag und den Anforderungen des $\S 56$ Abs. 2 Satz 1 SGB (Sozialgesetzbuch) VII unter den gegebenen Umständen gerecht zu werden, hat die multiprofessionell zusammengesetzte Expertengruppe ihr vielfältiges Fachwissen über Funktionsstörungen und deren Auswirkungen im Erwerbsleben zugrunde gelegt und darauf aufbauend neue MdEEckwerte konsentiert, die jeweils differenziert hergeleitet und begründet wurden. Die Vorschläge der Expertengruppe sollen zu einem gesellschaftlich, juristisch und sozialverträglichen Konsens führen und damit auch gewährlisten, dass die Unfallrente (MdE) auch zukünftig ihrer wichtigen Rolle als sozialem Befriedungsfaktor gerecht wird.

\section{Schlüsselwörter}

Reformbedarf · Amputation · Sozialgesetzbuch VII · Gliedmaßenverlust . Funktionsstörung

\section{Reduction in earning capacity (MdE) in the German statutory accident insurance. State of current discussion and results of the expert group}

\section{Abstract}

The expert group under the auspices of the German statutory accident insurance (DGUV) comprehensively, systematically and scientifically analyzed reduction in earning capacity (MdE) values for limb loss in an interdisciplinary methodological approach to review their relevance. The basis for the proposals of new MdE values is the International Classification of Functioning Disability and Health (ICF) related health and functional disorders that resulted in a grouping of comparable handicaps. The study examined which limitations on the labor market can be assumed today for insured patients with specific accident-related functional restrictions. For this purpose, a scientific literature search and a survey of the relevant occupational science institutes of the Federal Republic of Germany were carried out. As a result, however, it was not possible to generate valid labor market data on employment opportunities related to specific physical disorders. In order to fulfill the mandate and the requirements of $\S 56$ (2) sentence 1 of the Social Security Statutes VII (SGB VII) under the given circumstances, the multiprofessional group of experts applied its interdisciplinary knowledge on dysfunctions and effects on working world and developed new MdE benchmarks, which were each derived, differentiated and justified. The proposals of the expert group should lead to a socially, legally and politically acceptable consensus and thus guarantee that the accident benefits based on the MdE will continue to fulfill their important role as a factor for social satisfaction.

Keywords

Reform needs · Amputation · Social Security Statutes VII - Loss of limbs · Functional disorder 


\section{Methodisches Vorgehen der MdE-Expertengruppe}

Die Expertengruppe hat zunächst evaluiert, inwieweit sich aus wissenschaftlichen Veröffentlichungen bzw. arbeitsmarktspezifischen Quellen Erkenntnisse über die Leistungs- und Beschäftigungsfähigkeit von Menschen mit Amputationen ableiten lassen. Für diese Untersuchung wurde ein prototypischer Versicherter mit einer unfallbedingten Unterschenkelamputation und einer guten, Hilfsmittelversorgung, wie sie im Durchschnitt der Versorgungsrealität zu erwarten ist, definiert. Bei einer systematischen Literaturrecherche zu Aktivitäten und Teilhabe nach Unterschenkelamputation konnten aus 349 international publizierten Arbeiten am Ende 18 Publikationen eingeschlossen werden. Daraus konnten einige Hinweise für die Leistungsfähigkeit identifiziert werden, die im Wesentlichen auch schon in die S2kLeitlinie [7] zur Rehabilitation nach Major-Amputationen der unteren Extremität Eingang gefunden haben:

Die transtibiale Amputation ermöglicht die Nutzung des eigenen Kniegelenkes. Im Rahmen einer Unterschenkelkurzprothesenversorgung ist in Bezug auf die Berufswahl mittelschwere körperliche Tätigkeit zumutbar, die Tätigkeit kann in allen körperlichen Lagen verrichtet werden, wobei häufiges Arbeiten in der Hocke und in $Z$ wangshaltungen vermieden werden sollte.

Es fanden sich zudem weitere Kontextfaktoren von erheblichem Einfluss. Neben der Qualität der Versorgung und der Rehabilitation waren auch ein angemessener Arbeitsplatz bzw. die Möglichkeit zur Adaption des bestehenden Arbeitsplatzes von Bedeutung, insbesondere aber auch das Lebensalter und damit die individuelle Anpassungsfähigkeit des Versicherten.

Unter Rückgriff auf die in der Literaturrecherche gewonnenen Erkenntnisse und unter Beiziehung des Erfahrungswissens der beteiligten Mediziner und Rehabilitationswissenschaftler wurde ein positives und ein negatives Leistungsbild $\mathrm{zu}$ diesem prototypischen Versicherten entwickelt, mit einem für einen durch- schnittlich Versicherten zu erwartenden Aktivitätsniveau. Dieses Leistungsbild wurde dann an die Bundesanstalt für Arbeitsschutz und Arbeitsmedizin (BAuA) bzw. an das Bundesinstitut für Berufsbildung (BIBB) sowie an das Institut für Arbeitsmarkt- und Berufsforschung (IAB) mit folgender Fragestellung gerichtet:

Wie groß ist der prozentuale Anteil der Tätigkeiten des allgemeinen Arbeitsmarktes, welcher der versicherten Person unter Berücksichtigung des o.g. Restleistungsvermögens verschlossen ist (ohne Berücksichtigung des beruflichen Werdegangs und sonstiger berufsbezogener Qualifikationen und unabhängig von der Verweisbarkeit)?

Das Ergebnis der Anfrage war, dass keine der Datenbanken, die dem IAB, der BAuA/BIBB sowie der Bundesagentur für Arbeit zur Verfügung stehen, das von uns definierte Leistungsbild berücksichtigen und dass dazu auch keine analogen Arbeitsmarktdaten erhoben werden können. Die dem IAB zur Verfügung stehenden Datenbanken bilden keine ausreichend valide Grundlage für rechtlich fundierte Aussagen im Sinne einer medizinischen Eignungsfeststellung. Damit sind die Daten des IAB bzw. der Bundesagentur für Arbeit (BA) für die Zwecke der Arbeitsmarktanalyse bei der MdEEinschätzung ungeeignet.

Gleiches gilt für die von der $\mathrm{BiBB} /$ BAuA durchgeführte Erwerbstätigenbefragung [8]. Es handelt sich hierbei ausschließlich um subjektive Einschätzungen der Beschäftigten, die keinen Rückschluss auf die objektive Situation, d.h. die tatsächlichen Belastungen am Arbeitsplatz zulassen. Wenn überhaupt eine Aussage über konkrete krankheitsbedingte Einschränkungen getroffen werden kann, so müssten ganz erhebliche Selektionen bei der Gruppe der Befragten vorgenommen werden, die dann aufgrund der nur geringen Fallzahl keine Hochrechnung auf den allgemeinen Arbeitsmarkt in Deutschland mehr zulassen. Auch die BiBB/BAuA sieht deshalb ihre Studien in einem offiziellen Statement als für unsere Zwecke ungeeignet an.
Damit ist festzustellen, dass der methodische Ansatz, MdE-Werte anhand empirischer Daten zu definieren, nicht gelungen ist. Weitere Ansätze, um dies über andere wissenschaftliche Ansätze, ausgehend von den vorhandenen Arbeitsmarktdaten (Berufenet) und den darin enthaltenen Berufsinformationen $\mathrm{zu}$ versuchen, haben nach Rücksprache mit der IAB aus verschiedenen Aspekten heraus kaum Aussicht auf Erfolg.

\section{Bildung neuer MdE-Eckwerte}

Der Ansatz, die MdE-Werte in erster Linie mittels empirischer Daten mit belastbarem Bezug zum Arbeitsmarkt bzw. zum prozentualen Anteil der verschlossenen Erwerbsmöglichkeiten $\mathrm{zu}$ bestimmen, hat sich aufgrund der derzeit zur Verfügung stehenden Quellen als nicht umsetzbar erwiesen. Selbst wenn dies gelänge, so wäre dennoch ein nach geltender, höchstrichterlicher Rechtsprechung wichtiger Bestandteil der MdE-Einschätzung unberücksichtigt geblieben. Es handelt sich hierbei um den sog. immateriellen Schadensanteil, gekennzeichnet durch den Verlust der körperlichen Integrität, Entstellungen, Mehraufwand, Schmerzen etc. Diese Anteile an der MdE können aber nicht einfach berechnet werden, sondern sind als Ausfluss eines gesellschaftlichen Konsenses nach medizinischen, juristischen, sozialen und wirtschaftlichen Gesichtspunkten wertend zu berücksichtigen.

Um den Anforderungen des $\$ 56$ Abs. 2 Satz 1 SGB VII unter den gegebenen Umständen dennoch gerecht zu werden, hat die Expertengruppe im weiteren Verlauf v. a. auf das durch die Gruppe repräsentierte Erfahrungswissen über den allgemeinen Arbeitsmarkt, die Besonderheiten der verschiedenen Arbeitsbereiche in Groß-, Mittel- und Kleinunternehmen, die berufskundlichen Fachkenntnisse über physische und psychische Anforderungen sowie sozialmedizinische Rahmenbedingungen zurückgegriffen. Besonders hilfreich war in diesem Zusammenhang, dass die Gruppe nicht nur das traditionell wichtige orthopädisch-unfallchirurgische Wissen für Funktionseinschränkungen nach Amputationsverletzungen verkör- 


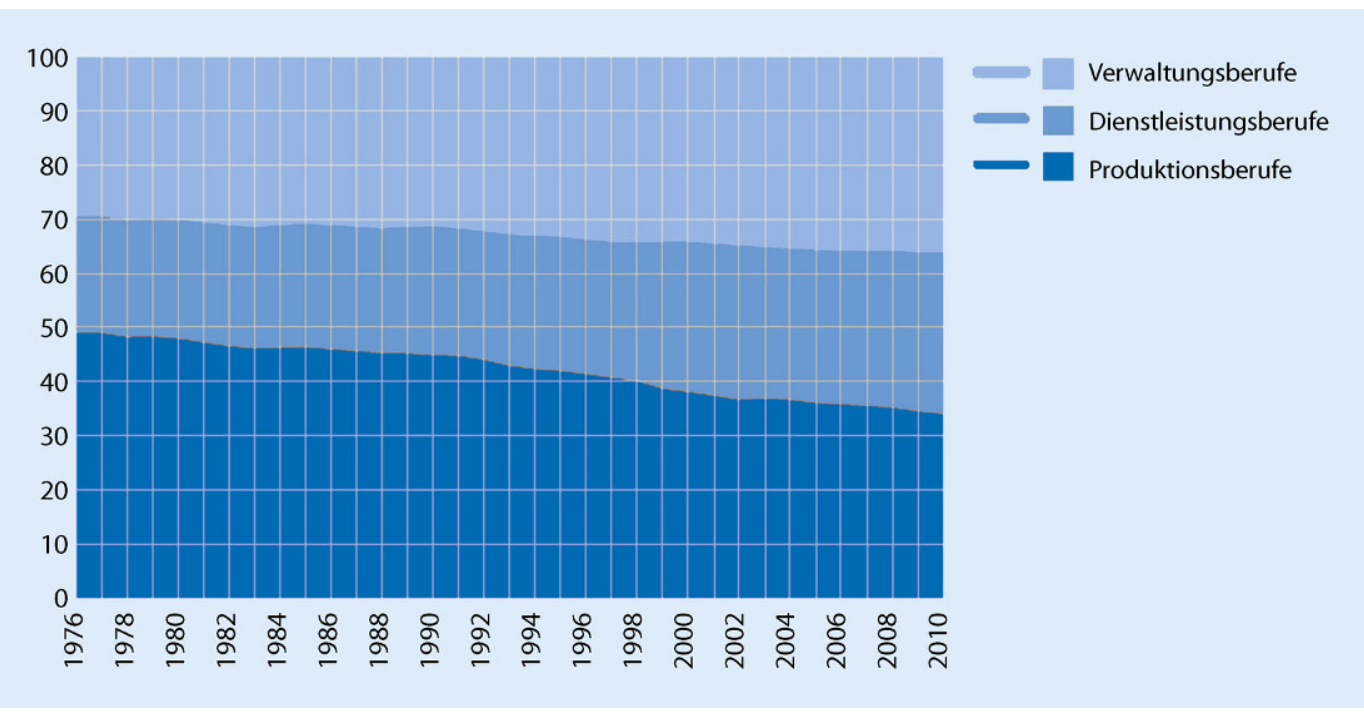

Abb. $1 \triangleleft$ Zeitliche, sektorale Entwicklung der Beschäftigungszahlen in den Hauptsektoren Verwaltung, Dienstleistung und Produktion. (Quelle: IAB Kurzbericht 9/2014, Abb. 4 [9])

perte, sondern dass darüber hinaus auch Expertenwissen $\mathrm{zu}$ den Fachgebieten Arbeitsmedizin, Sozialmedizin, Rehabilitationsmedizin, Berufskunde und Arbeitsmarkt vorhanden war. Auch wenn aus der Befragung der oben genannten Institutionen keine unmittelbaren empirischen Daten ableitbar sind, so geben deren Veröffentlichungen und die Befragung aber durchaus Hinweise, wie sich der Arbeitsmarkt und die Anforderungen an Beschäftigte über die letzten Jahrzehnte entwickelt haben. Beispielhaft seien hier die Berichte des IAB genannt [9], aus denen sich seit Mitte der 1970erJahre ein Rückgang der klassischen Produktionsberufe um ca. $15 \%$ von $50 \%$ auf ca. $35 \%$ ableiten lässt. Zugleich ist eher geringfügig der Verwaltungssektor um ca. $7 \%$, aber v. a. der Dienstleistungssektor um ca. $10 \%$ auf insgesamt mehr als $30 \%$ angewachsen ([9]; - Abb. 1).

Gerade in den Dienstleistungsberufen sind aber Flexibilität, äußere Erscheinung und Mobilität unter wechselnden bzw. vielfältigen Anforderungen von besonderer Bedeutung.

Die MdE-Expertengruppe hatte sodann die Chance ergriffen, im Rahmen eines sog. Delphi-Verfahrens die bisher in der Kritik stehenden Tabellenwerte und deren Uneinheitlichkeit zu überarbeiten. Bei diesem Delphi-Verfahren wurde der MdE-Expertengruppe eine Auflistung aller gängigen, zur gutachtlichen Standardliteratur gehörigen MdE-Tabellenwerte nach Funktionsstörung zur Verfügung gestellt, in mehreren Runden wurden zunächst anonym, dann mit vorgetragener Begründung einzelne Eckwerte identifiziert und konsentiert. Ausgehend von diesen, wurden dann weitere Funktionscluster mit zugehörigen MdE-Werten gebildet, die dann Eingang in ein einheitliches Tabellenwerk gefunden haben.

In diesem gruppeninternen Konsensusprozess wurden alle wichtigen Faktoren berücksichtigt und insbesondere auch ein Quervergleich mit den bisherigen und neuen MdE-Eckwerten anderer Verletzungsfolgen gezogen. Da die Unfallrente (MdE) v. a. eine Funktionsbeurteilung erfordert, erfolgte die Neueinschätzung der medizinischen Kriterien in Anlehnung an das biopsychosoziale Modell und die Grundlagen der ICF. Ausgehend von den schwersten Verletzungsfolgen wurden sog. Funktionsstörungsklassen gebildet. Dies ermöglicht einen Vergleich unterschiedlicher Bereiche und damit einen aus Sicht der MdEExpertengruppe nachvollziehbaren und begründbaren Neuansatz. Für die einzelnen Klassen bzw. Eckwerte werden lediglich Abstufungen von mindestens $10 \%$ gemacht, wenn sich im Vergleich mit anderen Eckwerten eine wesentliche Funktionsverbesserung bzw. Verschlechterung ergibt. Auf die Einschätzung von $5 \%$-Werten wird auch deshalb verzichtet, da diese wissenschaftlich abstrakt nicht begründbar sind. Selbstverständlich lässt es das SGB VII (anders als die VersMedV) auch weiterhin $\mathrm{zu}$, eine Unfallrente $\mathrm{z}$. B. von $35 \%$ einzuschätzen. Dies kann jedoch nur durch den Gutachter in Ansehung des konkreten Probanden im Einzelfall erfolgen. Die erzielten Ergebnisse wurden zudem in Bezug zu den neuen Werten des sozialen Entschädigungsrechts $(\mathrm{GdB} / \mathrm{GdS})$ gestellt und daraufhin überprüft, ob sich bei gleicher Verletzungsfolge im Hinblick auf die Teilnahme am Leben in der Gemeinschaft (GdB/GdS) allein bezogen auf den Arbeitsmarkt (MdE) ggf. eine stärkere oder schwächere Ausprägung der Einschränkungen ergibt.

\section{Gemeinsame Grundsätze zur MdE}

Die vorgeschlagenen MdE-Eckwerte beruhen auf einer bestmöglichen Versorgung, insbesondere im Hinblick auf

- funktionelle Kompensation und Rekonstruktion,

- Stumpfqualität, Muskelmantel,

- Nervenfunktionen,

- Narbenbildung,

- Hilfsmittelversorgung.

„Bestmöglich“ meint dabei nicht eine theoretisch bestmögliche Versorgung, d.h. außergewöhnlich gute Ergebnisse (z.B. bei prothetisch an Unterschenkel oder Oberschenkel vorsorgten Leistungssportlern) sind nicht berücksichtigt. Entscheidend ist das regelhaft bei dem überwiegenden Anteil der Patienten erreichbare Therapieergebnis auf 
Tab. 1 MdE(Minderung derErwerbsfähigkeit)- und GdS(Grad der Schädigungsfolge)/GdB(Grad der Behinderung)-Eckwerte im Vergleich verschiedener Gliedmaßenverluste

\begin{tabular}{|l|l|l|l|l|}
\hline Gliedmaßenverlust & $\begin{array}{l}\text { MdE } \\
\text { bisher }\end{array}$ & $\begin{array}{l}\text { Ludolph/ } \\
\text { Schürmann }\end{array}$ & $\begin{array}{l}\text { GdS/GdB } \\
\text { Neu } \\
\text { (bisher) }\end{array}$ & $\begin{array}{l}\text { MdE neu } \\
\text { Experten- } \\
\text { gruppe }\end{array}$ \\
\hline Arm im Oberarm oder Ellenbogen & 70 & 50 & 70 & 70 \\
\hline Hand & 60 & 45 & $60(50)$ & 60 \\
\hline Daumen & 20 & 25 & $30(25)$ & 30 \\
\hline Bein im Hüftgelenk & 80 & 60 & 80 & 80 \\
\hline Bein im Oberschenkel (Kurzstumpf) & 70 & 40 & 70 & 70 \\
\hline Bein im Unterschenkel & 40 & 30 & 40 & 40 \\
\hline
\end{tabular}

Grundlage leitliniengerechter Therapie. Im Rahmen der Hilfsmittelversorgung schließt dies die Versorgung mit einer modernen Funktionsprothese ein.

Eine funktionelle Rekonstruktion bzw. optimale Hilfsmittelversorgung (Prothetik) kann die Funktionsbeeinträchtigung nur teilweise ausgleichen. Nicht kompensiert wird die bei Amputation (insbesondere) größerer Gliedmaßen fortbestehende Gesundheitsstörung, die regelhaft zu einer erheblichen Beeinträchtigung des äußeren Erscheinungsbildes sowie der körperlichen Integrität führt und auch eine erhebliche Beeinträchtigung der menschlichen Interaktion bedingen kann (so z. B. auch bei Verbrennungen, Entstellungen etc.). Diese Auswirkungen wurden in Abhängigkeit von der Schwere der Gesundheitsstörung bei der Festsetzung der MdEWerte als immaterieller Schadensanteil mit bewertet. Berücksichtigt wurden zudem die durch den Extremitätenverlust bedingte Fehlwahrnehmung (Phantomgefühl) sowie die damit regelmäßig in Verbindung stehenden Schmerzen, soweit sie mit dem feststellbaren Ausmaß der Gesundheitsstörung korrelieren.

Auswirkungen auf die Erwerbsfähigkeit und somit auf die Höhe der MdE haben insbesondere Einschränkungen bzw. Störungen

- der Bewegungsfreiheit, Mobilität, Fortbewegung,

- der Benutzung von Transportmitteln und Fahrzeugen,

- des sicheren Standes bzw. Überwindung von Steigungen, Unebenheiten,

- der sensiblen Empfindung, des Tastsinns, der Feinmotorik, des Greifens,
- des Tragens und Handhabens von Lasten sowie Druck- und Kraftausübung,

- der Selbstversorgung,

- des Schreibens, der PC-Handhabung,

- der nonverbalen Kommunikation (Ausdrucksfähigkeit, Gestik),

- der Leistungsfähigkeit in Bezug auf übliche Arbeitszeiten und der damit verbundenen Beanspruchungen.

\section{Auszugsweise Begründungen obere und untere Gliedmaßen}

Den oberen Gliedmaßen kommt aufgrund der besonderen Bedeutung manueller Tätigkeiten im Arbeitsleben eine zentrale Stellung zu, was insbesondere bei der Gegenüberstellung von Hand/ Finger zu Fuß/Zehen deutlich wird. Distale Armwerte sind wegen der Bedeutung manueller Funktionen deshalb generell höher einzuschätzen als distale Beinwerte. Dabei sind v. a. die Funktionen der Fein- und Grobmotorik, der Sensibilität und Gestik mit berücksichtigt. Auch die Beeinträchtigung der körperlichen Integrität ist im oberen Gliedmaßenbereich aufgrund der vermehrten Sichtbarkeit des Körperschadens besonders hoch ausgeprägt.

Von herausragender Bedeutung für die Handfunktion ist der Daumen. Insbesondere die Oppositionsbewegung des Daumens ermöglicht es erst, bestimmte wichtige Greiffunktionen auszuführen. Bei Verlust des Daumens im Grundgelenk (MdE $30 \%$ ) stellt der zusätzlich zum Daumen eintretende Verlust nur eines Langfingers noch keine wesentlich andere (zusätzliche) Beeinträchtigung dar und verursacht deshalb keine höhere MdE.
Auch wenn den unteren Gliedmaßen im heutigen Arbeitsleben grundsätzlich eine geringere Bedeutung zukommt, so sind die dauerhafte Belastungsfähigkeit sowie ausreichende Mobilität und Flexibilität gerade in dem großen Segment der Dienstleistungsberufe von großer Relevanz für die Beschäftigung. Im Vergleich zum Verlust des Armes im Unterarm bzw. der Hand $(60 \%)$ wirkt sich der Verlust eines Beines im Unterschenkel auf die Erwerbsfähigkeit deshalb zwar geringer aus, ist aber dennoch weiterhin mit einer MdE von $40 \%$ einzuschätzen.

Für die oberen und unteren Gliedmaßen ergeben sich im Vergleich zu - den bisher ganz überwiegend vorgeschlagenen MdE-Werten in den unfallmedizinischen Standardwerken (Schönberger, Mertens und Valentin [10, S. 605 ff., 716 ff.]; Mehrhoff, Ekkernkamp und Wich [11, S. $168 \mathrm{ff}]$.$) ,$ s. - Tab. 1, MdE-Eckwerte Spalte 1 (MdE bisher),

- den von Ludolph und Schürmann et al. vorgeschlagenen MdE-Werten $[4,5]$, s. - Tab. 1, MdE-Eckwerte Spalte 2 (Ludolph/Schürmann ) - den aktuellen und angekündigten neuen GdB/GdS-Werten der Versorgungsmedizinverordnung (VersMedV), s. - Tab. 1, GdS/GdBEckwerte Spalte 3 (s. VersMedV bisher und VersMedV neu)

auszugsweise folgende reformierte MdEEckwerte im Überblick, s. • Tab. 1, MdEEckwerte Spalte 4 (MdE neu).

Die Ergebnisse des Konsensprozesses der MdE Expertengruppe sollen u.a. am 27.06.2018 in Berlin der Fachöffentlichkeit vorgestellt und zusammen mit Vertretern der Fachgesellschaften, Rechtsanwendern, sozialpolitischen Entscheidungsträgern, Betroffenenverbänden, Sozialpartnern und den Unfallversicherungsträgern diskutiert werden. Anschließend soll der finalisierte Konsensbericht mit Begründungen und den neuen Eckwerten bei Amputationen veröffentlicht werden.

\section{Fazit für die Praxis}

- Die MdE in der gesetzlichen Unfallversicherung ist Maßstab für 
die Höhe der Verletztenrente. Sie wird durch Bezugnahme auf in der Gutachtenliteratur veröffentlichte Erfahrungswerte individuell und fallbezogen eingeschätzt.

- Im Gesetzestext zur MdE findet sich ausschließlich ein Bezug zwischen unfallbedingter Funktionsstörung und den dadurch verwehrten Arbeitsmöglichkeiten des Verletzten. Die Rechtsprechung hat aber eine weitere Dimension der Unfallrente festgestellt, die aus der Zweckbestimmung der Rente sowie der Ablösung der Unternehmerhaftung resultieren (sog. immaterielle Schadensanteile).

- Auch die zukünftigen Tabellenwerke sollen in der Rechtsanwendung einen festen Platz finden und als Orientierungshilfe für die aktuelle Begutachtungsliteratur fungieren.

- Die MdE bzw. die Unfallrente muss auch zukünftig ihrer wichtigen Funktion als (sozialer) Befriedungsfaktor gerecht werden.

- Eine notwendige Harmonisierung und Abstimmung der vorhandenen Erfahrungswerte ist von der Expertengruppe in Gang gesetzt worden und soll in einem gesellschaftlichen Dialog zu einem sozial verträglichen Konsens führen.

\section{Korrespondenzadresse}

Ass. jur. D. Scholtysik

Spitzenverband der gewerblichen Berufsgenossenschaften und der Unfallversicherungsträger der öffentlichen Hand, Deutsche Gesetzliche Unfallversicherung (DGUV)

Glinkastr. 40, 10117 Berlin, Deutschland dirk.scholtysik@dguv.de

\section{Einhaltung ethischer Richtlinien}

Interessenkonflikt. D. Scholtysik ist Beschäftigter der DGUV. M. Wich ist Beratender Arzt der DGUV, BG der Bauwirtschaft und der BG Nahrungsmittel und Gastgewerbe.

Dieser Beitrag beinhaltet keine von den Autoren durchgeführten Studien an Menschen oder Tieren.

The supplement containing this article is not sponsored by industry.

\section{Literatur}

1. Nusser A, Spellbrink W (2017) Die Rechtsnatur der MdE-Tabellen im Recht der Gesetzlichen Unfallversicherung, Die Sozialgerichtsbarkeit SGb 10.17, S550

2. Deutsche gesetzliche Unfallversicherung (DGUV) (2016) DGUV Statistiken für die Praxis. http://publikationen.dguv.de/dguv/pdf/10002/ 12639neu.pdf.Zugegriffen:26. März 2018

3. Schürmann J (2014) MdE nach Hilfsmittelversorgung oder Medikation. Trauma Berufskr 16:204-210

4. Ludolph E, Schürmann J (2016) Neubewertung der MdE bei unfallchirurgisch-orthopädischen Arbeitsunfall- und BK-Folgen in der gesetzlichen Unfallversicherung (GUV). MedSach 112(2):60-71

5. Ludolph E, Schürmann J, Gaidzik PW (Hrsg) (2017) Kursbuch der ärztlichen Begutachtung. Überarbeitung der MdE-Erfahrungswerte auf unfallchirurgisch-orthopädischen Gebiet (GUV). Ecomed, Landsberg (Kap. III-1.10.5)

6. Jung HP (2013) Sitzung der Kommission SGB VII des Deutschen Sozialgerichtstags e. V. am 15.11.2012 in Potsdam. Trauma Berufskr 15:283-290

7. AWMF Online (2013) Leitlinien-Detailansicht Rehabilitation nach Majoramputation an der unteren Extremität (proximal des Fußes). http:// www.awmf.org/leitlinien/detail//l/033-044.html Zugegriffen:26. März 2018

8. Bundesanstalt für Arbeitsschutz und Arbeitsmedizin (BAuA) (2012) Grundauswertung der BAuABIBB-Erwerbstätigenbefragung. BAuA, Dortmund. http://www.baua.de/de/Publikationen/ Fachbeitraege/Gd73.Zugegriffen: 26. März 2018

9. Institut für Arbeitsmarkt- und Berufsforschung (IAB) (2014z) Kurzbericht 9/2014 zur Verteilung der Beschäftigten auf die Sektoren (Abb. 4). http://doku.iab.de/kurzber/2014/kb0914.pdf. Zugegriffen:26. März 2018

10. Schönberger A, Mehrtens G, Valentin H (2017) Arbeitsunfall und Berufskrankheit, 9. Aufl. Erich Schmidt, Berlin

11. Mehrhoff F, Ekkernkamp A, Wich M (2012) Unfallbegutachtung, 13. Aufl. de Gruyter, Berlin 